\title{
CONSTRUÇÃO DE MOSAICOS TEMPORAIS NORMALIZADOS DE IMAGENS PLANET
}

\author{
Marciano Saraiva ${ }^{1}$, Diego Silva ${ }^{2}$, Luiz Ferreira ${ }^{3}$, Soltan Galano ${ }^{4}$, João Siqueira ${ }^{5}$ Carlos Souza Jr. ${ }^{6}$ \\ ${ }^{1}$ Agrosatélite Geotecnologia Aplicada, 88032-005, Florianópolis - SC, Brasil, marciano@agrosatelite.com.br \\ ${ }^{2}$ Terras App Solutions, 66060-021, Belém - PA, Brasil, diego.silva@terras.agr.br \\ ${ }^{3}$ Solved - Soluções em Geoinformação, 66075-750, Belém - PA, Brasil, luiz.cortinhas@solved.eco.br \\ ${ }^{4}$ DMMDC/UFBA, 40110-909, Salvador - BA, Brasil, solkan1201@gmail.com \\ ${ }^{5}$ JVN Siqueira - ME, 66060-021, Belém - PA, Brasil, joaovsiqueira1@gmail.com \\ ${ }^{6}$ IMAZON - Instituto do Homem e Meio Ambiente, 66055-200, Belém - PA, Brasil, souzajr@imazon.org.br
}

\section{RESUMO}

A constelação de satélites compactos Planet coleta imagens multiespectrais diariamente com resolução espacial de 3 a 4 $\mathrm{m}$. Essas imagens têm um enorme potencial para contribuir com a melhoria da precisão do mapeamento do uso e da cobertura do solo, e na deteç̧ão e monitoramento de interferências antrópicas na paisagem. Uma etapa fundamental para o uso efetivo de imagens Planet nesses tipos de aplicações é o seu pré-processamento para detecção e remoção de nuvens, sombra e outros tipos de ruídos atmosféricos e a normalização radiométrica de séries temporais de mosaicos. Neste artigo, apresentamos os avanços do Projeto NextGenMap para produzir mosaicos de imagens Planet para diversas aplicações. Apresentamos o algoritmo detalhado, o acesso aos códigos implementados na Plataforma Google Earth Engine e o repositório dos mosaicos. Por último, discutimos os próximos passos para superar algumas das limitações que ainda não foram superadas nessa fase do projeto.

Palavras-chave - Imagens Planet, Earth Engine, Remoção de Nuvens, Normalização

\section{ABSTRACT}

The compact multispectral satellite constellation, Planet, collects daily images with a spatial resolution of 3 to $4 \mathrm{~m}$. These images have the potential to contribute significantly to the improvement of land use and land cover mapping accuracy and to enhance the detection and monitoring capabilities of anthropogenic interferences over natural landscapes. A fundamental step for the effective use of Planet's images is the detection and removal of clouds, shades and other types of atmospheric noise, plus the radiometric normalisation of such dataset. In this article, we briefly present the Nextgen Map Project, as well as, share its advances towards the generation of normalised cloud-free mosaics. In here, the Google Earth Engine algorithm is detailed presented, and the mosaic final repository is shared. Finally, we discuss the next steps to overcome some of the limitations that have not yet been addressed in this phase of the project.

Keywords - Planet Imagery, Earth Engine, Cloud Removal, Normalization

\section{INTRODUÇÃO}

Imagens multiespectrais da constelação dos satélites Dove $(+130)$ da empresa Planet coletam diariamente imagens PlanetScope com resolução espacial de 3-4 m. Essas imagens são adquiridas em quatro bandas espectrais (Blue: 455 - $515 \mathrm{~nm}$, Green: 500 - $590 \mathrm{~nm}$, Red: $590-670 \mathrm{~nm}$, NIR: $780-860 \mathrm{~nm}$ ) e estão disponíveis em diferentes modos de pré-processamento. Os satélites estão posicionados em duas órbita, $400 \mathrm{~km}$ (Estação Espacial Internacional) e heliossíncrona de $475 \mathrm{~km}$, com hora local solar entre 9:30 e 11:30 [1]. Essas imagens oferecem um grande leque de aplicações de sensoriamento remoto. Contudo, há um desafio de como processar imagens PlanetScope em larga escala temporal e espacial para obter imagens normalizadas. Neste artigo, apresentamos o protocolo de pré-processamento de imagens PlanetScope do projeto NextGenMap.

O projeto NextGenMap é uma iniciativa multi-institucional envolvendo Universidades, ONGs e empresas da área geoespacial, com o objetivo geral de desenvolver algoritmos de aprendizagem de máquina, ferramentas e métodos para produzir mapas atualizados, detalhados e precisos da cobertura e uso da terra, e para o monitoramento do desmatamento, restauração e de mudanças da cobertura terrestre. Os objetivos específicos do projeto NextGenMap são: i) tornar operacional o uso de imagens de alta resolução espacial e temporal para aplicações de larga escala, ii) desenvolver métodos eficientes, ferramentas e algoritmos para processar e extrair informações dessas imagens, e iii) desenvolver a linha de produção para detectar desmatamento, degradação da terra, restauração e de outros usos. Nesta fase inicial do projeto tivemos acesso a imagens PlanetScope no período de 1 de agosto de 2017 a 30 de novembro de 2018, cobrindo uma área total de $526.400 \mathrm{~km}^{2}$. 
Neste artigo, apresentamos o protocolo de pré-processamento das imagens diárias PlanetScope para criação de mosaicos temporais nas escalas semanal, quinzenal e mensal. Esse protocolo inclui a geração de uma máscara de nuvens e sombra, da normalização radiométrica e a criação dos mosaicos. Além disso, apresentamos a estrutura computacional para o processamento desse conjunto de dados integrado a Plataforma Google Earth Engine, bem como os códigos para o pré-processamento e os repositórios dos mosaicos processados. Por último, avaliamos a qualidade dos mosaicos e discutimos as aplicações e os próximos passos para melhoria do algoritmo.

\section{MATERIAIS E MÉTODOS}

\section{1 Área de Estudo}

A área de estudo do projeto NextGenMap está distribuída nos seis biomas brasileiros (Amazônia, Caatinga, Cerrado, Mata Atlântica, Pampa, Pantanal) e no bioma Chaco, localizado entre os países da Argentina, Paraguai e Bolívia. Ao todo, através do sistema Internacional ao Milionésimo, foram definidas 16 cartas, na escala 1:250.000 (Figura 1). Cada carta cobre uma área de aproximadamente $37.600 \mathrm{~km}^{2}$.

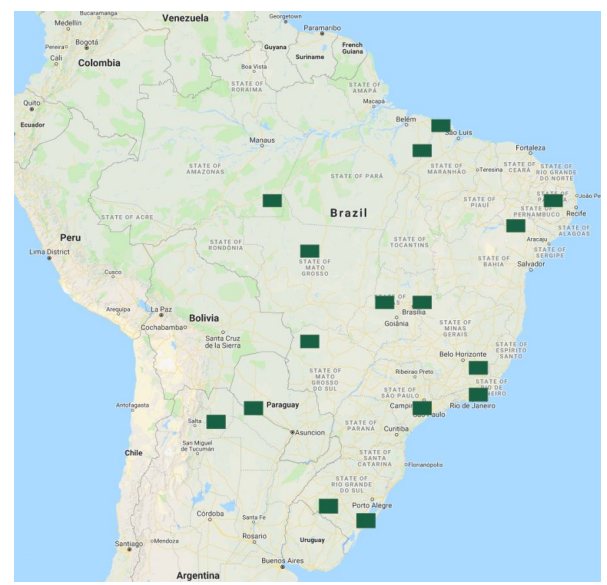

Figura 1. Cobertura de imagens Planet.

\subsection{Imagens Planet}

Foram utilizadas as imagens Planet no formato Analytic PlanetScope Ortho Scenes. Essas imagens foram adquiridas de satélites que estão em duas órbitas: i) da Estação Espacial Internacional, a 400 km com 51,6 graus de inclinação; e ii) heliossíncrona com inclinação quase-polar de 98 graus [1].

\subsection{Pré-processamento}

Inicialmente, foi realizada a conversão da coleção de imagens de radiância (L $\lambda)$ para reflectância $(\rho \lambda)$ no topo da atmosfera (TOA). A reflectância TOA é calculada de acordo com a equação [2]:

$$
\rho_{\lambda}=\left(\pi \times L_{\lambda} \times d^{2}\right) /\left(E S U N_{\lambda} \times \cos \theta_{s}\right) \quad(\text { eq } 1)
$$

Onde: $\rho_{\lambda}=$ reflectância planetária no topo da atmosfera [adimensional], $\pi=$ constante matemática igual a $\sim 3,14159$ [adimensional], $L_{\lambda}=$ radiância espectral no sensor $[\mathrm{W} / \mathrm{m} 2$ sr $\mu \mathrm{m}$ ], $\mathrm{d}=$ distância Terra-Sol [unidade astronômica], $E S U N_{\lambda}=$ irradiância solar estratosférica média $[\mathrm{W} / \mathrm{m} 2$ $\mu \mathrm{m}] \mathrm{e}$

$\theta_{s}=$ ângulo zenital Solar [graus].

Os valores adotados de $E S U N_{\lambda}$ foram 1997, 1812, 1533 e 1039, correspondentes às quatro bandas espectrais Blue, Green, Red e NIR das imagens PlanetScope, respectivamente. Esses foram obtidos por aproximações do sensor ETM+ do programa Landsat.

A partir da coleção de imagens em reflectância TOA, foi gerado um mosaico de referência e uma coleção de imagens intercalibração ao mosaico de referência. O mosaico de referência é construído a partir da mediana da coleção de imagens com percentual de cobertura de nuvens menor que 1\%. Esta informação está contida no metadado de cada imagem Planet. Todas as imagens do período (que cobre de agosto de 2017 até 30 de novembro de 2018) da coleção foram utilizadas para compor esta imagem de mediana.

A coleção de imagens é inter-calibrada usando o mosaico de referência para ajustar o histograma das bandas de cada imagem. Para reduzir a quantidade de ruídos no mosaico final, optou-se pela seleção das imagens com percentual de cobertura de nuvens abaixo de 20\%. Em seguida, fazendo uma iteração por banda da imagem de referência e de cada imagem de entrada, são gerados os histogramas para os valores e frequências dos dados. Com os valores de frequência obtidos é calculada a função de distribuição cumulativa (cumulative distribution function CDF) e então foi realizada a normalização dos valores dessa função, baseado na divisão de cada valor pelo valor total da CDF. O ajuste dos histogramas utiliza esses dados para criar dois regressores estatísticos baseados no Random Forest [4], um para a imagem de referência e um para a imagem de entrada. Os regressores estatísticos são utilizados para prever os valores de reflectância, produzindo uma classificação para cada imagem, tanto da referência quanto da imagem à ser ajustada. Essa estratégia usa a árvore de regressão (produto dos regressores estatísticos Regression Tree) para predizer valores de pixels sob nuvens e sombras de nuvem [3].

Em seguida, são aplicadas as máscaras de nuvens e sombra para cada imagem normalizada, ver item 2.4. Após aplicação da máscara é calculada a estatística de mediana 
para o conjunto de pixels não mascarados. Em paralelo a etapa de remoção de sombra e nuvens é executado um regressor estatístico baseado em Random Forest, para reduzir ruídos entre infraestrutura urbana e água, devido a semelhanças espectrais, não reconhecidas pela máscara. Ao final, os mosaicos normalizados e livres de nuvens e sombras são exportadas em formato Geotiff.

Os mosaicos exportados incluem as bandas espectrais Blue, Green, Red, NIR, e uma banda (Availability) indicando quantas imagens foram usadas no mosaico. Um metadado contendo informações de todas as imagens utilizadas, percentual de nuvens e do período selecionado é adicionado ao mosaico, ver Figura 2.

\subsection{Máscaras para Remoção de Sombras e Nuvens}

O algoritmo para gerar as máscaras de nuvens e sombras se baseiam em valores empiricamente escolhidos para separação dos alvos por fatiamento do histograma. Para nuvens, foi escolhido o limiar de valores acima 2200 na banda Blue. Para a máscara de sombra, foram utilizados os valores entre 1500 e 2200 , respectivamente, para as bandas do Blue e NIR, a execução dos limiares resultam na máscara de sombras e nuvens.

A máscara de sombras e nuvens tem seu refinamento através da execução do filtro frequência. Esta etapa, consiste na avaliação dos alvos da máscara quanto a sua persistência temporal. A remoção de pixels estáveis atua sobre toda a série temporal, mantendo-se os pixels de baixa estabilidade temporal $(<50 \%)$, como Nuvens e Sombras, e eliminando pixels estáveis no tempo, casos de Corpos D'água, Praias, Construções Urbanas e Solo Exposto.

A etapa de Remoção de Nuvens e Sombras, opera em cada imagem, removendo os pixels mascarados e retornando para o fluxo de execução apenas imagens livres de nuvens.

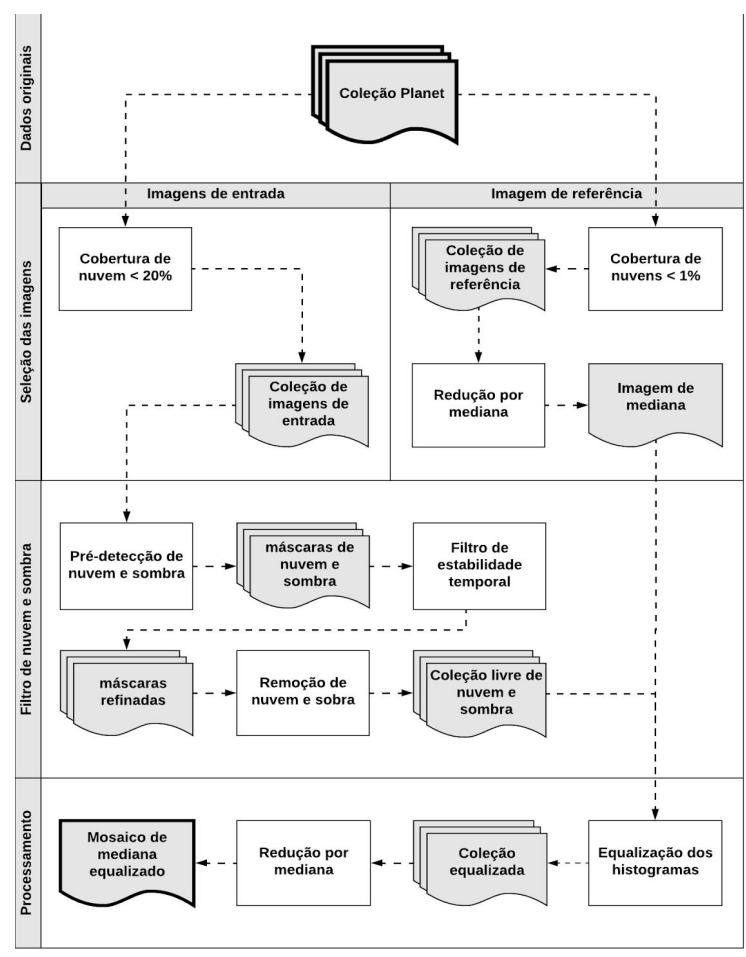

Figura 2. Fluxograma do pré-processamento de imagens Planet.

\section{RESULTADOS}

Foram produzidos 52 mosaicos semanais, 26 mosaicos quinzenais e 13 mosaicos mensais para o período de 1 de agosto de 2017 a 31 de agosto de 2018, para cada uma 16 cartas do projeto. As cartas SA-23-V-D, SB-24-Z-D e SC-24-V-D apresentaram alta cobertura e frequência de nuvens não permitindo gerar mosaicos de qualidade. Para as demais cartas, os mosaicos semanais tiveram uma cobertura média de 200 imagens, aproximadamente. Já para os mosaicos quinzenais a cobertura média de imagens Planet oscila de 320 à 340 imagens.

Os melhores resultados para a geração de mosaicos foram obtidos para o período mensal, segundo o critério de baixa cobertura de nuvens (20\%) e ausência de neblina.

Para as demais cartas $(n=13)$, os mosaicos de imagens produzidos com o algoritmo proposto apresentaram consistência espacial e temporal satisfatórias, ver Figura 3. 


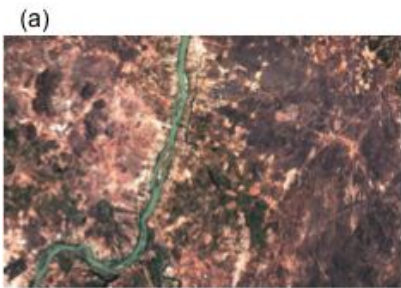

(c)
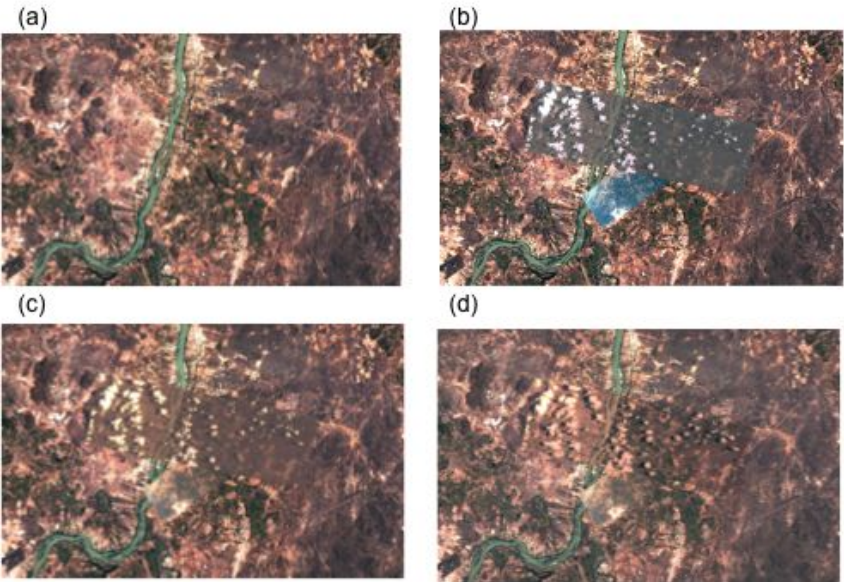

(d)

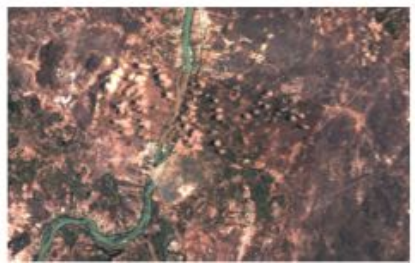

Figura 3. Exemplo de imagem de mosaico de referência Planet (a), com imagens não normalizadas (b), e normalizadas usando ajuste de histograma linear (c) e não-linear (d).

A consistência espectral também pode ser observada através da variação da média mensal do índice de vegetação NDVI. Para a Figura 4 abaixo, observa-se a variação do NDVI mensal para um ponto sobre um pivô central de irrigação na carta SD-23-Y-C.

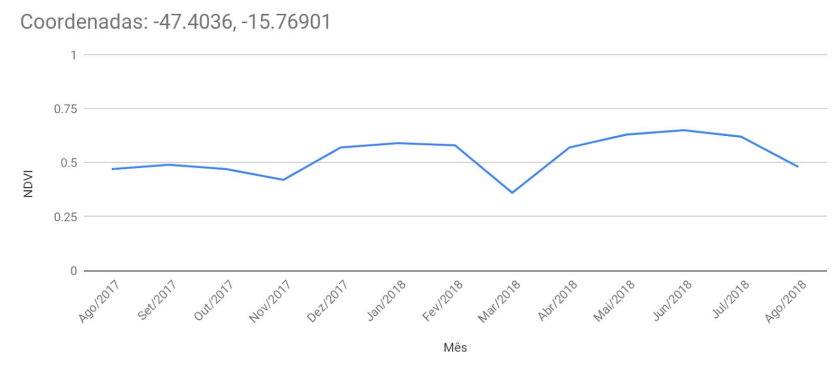

Figura 4. Série temporal média de NDVI

\section{DISCUSSÃO}

Durante o processo de refinamento das máscaras de sombras e nuvens, foram encontradas dificuldades na separação entre estes dois alvos quando não aplicado o filtro temporal. Causado por sombreamentos associados a altos valores de slope (diferenças de altitude).

Por outro lado, as sombras que não são persistentes no tempo, por exemplo, as de nuvens, foram em sua maioria mascaradas e removidos da imagem, evitando a inclusão de corpos de água à máscara. $\mathrm{O}$ filtro temporal aplicado a máscara de nuvens é importante para separação de alvos com alto valor de saturação como: praias, solo exposto, nuvem, e infraestrutura urbana; o filtro teve sucesso na separação dos alvos uma vez que as nuvens normalmente não possuem incidência contínua sobre a superfície terrestre.

As cartas do bioma Caatinga (SB-24-Z-D e SC-24-V-D) apresentaram alta contaminação de haze. Para estes casos, sugere-se a utilização dos índices Total Reflectance Radiance Index (TRRI)[5] e Cloud-Soil index (CSI)[6] capazes de apresentar maior resistência às variações espectrais associadas ao bioma Caatinga. Já está em andamento uma abordagem alternativa de eliminação de nuvens e sombras nestes casos, os resultados deste estudo devem ser apresentados em estudos posteriores.

\section{CONCLUSÕES}

Os produtos gerados demonstram a capacidade de processamento em larga escala temporal e espacial de imagens Planet. Mais além, os algoritmos desenvolvidos permitiram a obtenção de mosaicos radiometricamente normalizados. A partir de imagens diárias foi possível a criação de mosaicos em três diferentes escalas temporais; semanal, quinzenal e mensal. Postas juntas estas características podem permitir a aplicação de imagens planet em segmentos diversos do sensoriamento remoto como: sistemas de detecção de mudanças, sistemas de alerta em tempo real e mecanismos de aumento da acurácia de mapeamento. Por fim, deve-se ressaltar que em regiões que apresentem alta contaminação por hazes faz-se necessário a criação de outros métodos de eliminação de ruídos atmosféricos.

\section{REFERÊNCIAS}

[1] Planet, "PLANET IMAGERY PRODUCT SPECIFICATIONS.” p. 91, 2018.

[2] G. Chander, B. L. Markham, and D. L. Helder, "Summary of current radiometric calibration coefficients for Landsat MSS, TM, ETM+, and EO-1 ALI sensors," Remote Sens. Environ., vol. 113, no. 5, pp. 893-903, 2009.

[3] E. H. Helmer and B. Ruefenacht, "Cloud-Free Satellite Image Mosaics with Regression Trees and Histogram Matching," Photogrammetric Engineering \& Remote Sensing, vol. 71, no. 9. pp. 1079-1089, 2005.

[4] BARANDIARAN, Iñigo. The random subspace method for constructing decision forests. IEEE transactions on pattern analysis and machine intelligence, 1998, vol. 20, no 8.

[5] DUONG, N. D. (1998). Total reflected radiance index-An index to support land cover classification. In: Proc. Asian Conference on Remote Sensing (ACRS), Manila, Philippines, Vol. 7.

[6] ZHAO, S., \& DAI, S. (2016). Automated Cloud Removal and Filling in Optical Remote Sensing Images. In: Virtual Reality and Visualization (ICVRV), 2016 International Conference on. IEEE. pp: 292-297. 